Célia Dias dos Santos 1

Leonor Maria Pacheco Santos 2

José Natal Figueiroa ${ }^{3}$

Pajuçara Maria Guimarães Marroquim 1

Maria Alice Araújo Oliveira 1

\title{
Anemia em escolares da primeira série do ensino fundamental da rede pública de Maceió, Alagoas, Brasil
}

\author{
Anemia in public school first graders \\ in the city of Maceió, Alagoas, Brazil
}

1 Departamento de Nutrição, Universidade Federal de Alagoas. Campus A. C. Simões, BR 101/Norte, Km 14 Maceió, $A L$ 57072-970, Brasil. caiodias@hotlink.com.br 2 Departamento de Ciência e Tecnologia em Saúde, Secretaria de Politicas de Saúde, Ministério da Saúde. Esplanada dos Ministérios, Bloco G, Sala 116, Brasília DF 70058-900, Brasil.

leonor.pacheco@saude.gov.br

3 Instituto Materno Infantil de Pernambuco. Rua dos Coelhos 300 , Recife, $P E$

50070-550, Brasil.

\begin{abstract}
A cross-sectional study was conducted in a representative sample of 426 randomly selected first graders (ages 6 to 10 years) from public schools in Maceió, State of Alagoas, Brazil. The aim was to determine the prevalence of anemia, as well as its association with growth retardation. Data were collected from May to July 2000, and determination of hemoglobin (HGB) employed an STKS Coulter counter. Two cut-off points were used to classify anemia, both established by the World Health Organization: $H G B<11.5 \mathrm{~g} / \mathrm{dl}$ and $H G B<12.0 \mathrm{~g} / \mathrm{dl}$. The indicators height/age (H/A), weight/age (W/A), and weight/height (W/H) below -2.0 standard deviations from the NCHS reference were diagnosed as growth retardation. Prevalence of anemia was 9.9\% when $H G B<11.5 \mathrm{~g} / \mathrm{dl}$ was used, and $25.4 \%$ when the cut-off point was $H G B<12.0 \mathrm{~g} / \mathrm{dl}$. Growth retardation was detected in $6.2 \%$ of children according to $H / A, 4.0 \%$ for W/A, and $3.0 \%$ for $W / H$. There was no statistically significant association between the variables in the study. These findings confirm results of previous surveys where prevalence of anemia was much higher than that of growth retardation. The severe consequences of anemia in this age group justify the implementation of broad public policies to overcome this nutritional deficiency.
\end{abstract}

Key words Anemia; School Health; Cross-Sectional Studies

Resumo Realizou-se um estudo transversal com amostra probabilística de 426 alunos de 6 a 10 anos, da 1a série do ensino fundamental das escolas públicas de Maceió, Alagoas, Brasil. O objetivo foi avaliar a prevalência da anemia e sua associação com o retardo de crescimento. Os dados foram coletados entre maio e julho de 2000. O sangue foi colhido por venipuntura e a dosagem de hemoglobina ( $\mathrm{Hb}$ ) foi realizada pelo contador Coulter STKS. Classificou-se a anemia por dois critérios estabelecidos pela Organização Mundial da Saúde: $\mathrm{Hb}<11$,5g/dl e Hb<12,0g/dl. O retardo pondo-estatural foi diagnosticado quando os indicadores Altura/Idade (A/I), Peso/Idade (P/I) e Peso/Altura (P/A) se encontravam abaixo de -2,0 desvios-padrão da referência NCHS. A prevalência da anemia foi de 9,9\%, considerando-se $\mathrm{Hb}<11$,5g/dl e de 25,4\%, utilizando-se $\mathrm{Hb}$ $<12,0 \mathrm{~g} / \mathrm{dl}$. O retardo do crescimento foi detectado em 6,2\% segundo A/I, 4,0\% pelo indicador P/I $e$ 3,0\% em relação a P/A. Não houve associação estatisticamente significativa entre as variáveis do estudo. Estes resultados confirmam os observados em outras pesquisas: prevalência de anemia muito superior à prevalência do retardo pondo-estatural. Conclui-se que as graves conseqüências da anemia nesta faixa etária, justificam a implementação de políticas de largo alcance para o enfrentamento do problema.

Palavras-chave Anemia; Saúde Escolar; Estudos Transversais 


\section{Introdução}

Apontada como a carência nutricional de maior magnitude no mundo, a anemia afeta grandes contingentes populacionais nas diversas faixas etárias. Os autores em geral concordam quanto aos grupos biológicos de maior risco para desenvolver a anemia: crianças de seis meses a três anos de idade e gestantes, embora as mulheres em idade reprodutiva tenham, também, elevado risco de se tornarem anêmicas. O mesmo acontece com os escolares que, em função da fase de crescimento e desenvolvimento acelerados, são considerados um grupo de risco (Ferreira, 1998).

Foi recentemente publicado o Consenso Técnico do United Nations Administrative Committee on Coordination (atual United Nations System Chief Executives Board for Coordination) e Sub-Committee on Nutrition (atual Standing Committee on Nutrition) sobre a deficiência de ferro e anemia, confirmando a definição da anemia como a redução da taxa de hemoglobina circulante atribuída a condições patológicas, dentre as quais a deficiência de ferro é a mais comum (UNICEF/UNU/WHO/ MI, 1999). Esta mesma publicação avaliou que a anemia está presente numa população, caso a prevalência de hemoglobina, abaixo dos valores mínimos consensuais para cada faixa etária, seja maior do que $5 \%$, e estimou que, atualmente, a anemia e a deficiência de ferro afetam mais de 3,5 bilhões de indivíduos no mundo em desenvolvimento (UNICEF/UNU/WHO/ MI, 1999).

As repercussões da anemia sobre o organismo humano são bastante conhecidas. A anemia atinge o crescimento e o desenvolvimento físico e mental das crianças, acarretando sonolência, incapacidade de fixar a atenção e diminuição na acuidade mental, o que leva ao comprometimento do rendimento escolar. Nesta faixa etária pode ainda provocar redução da capacidade imunológica (acarretando maior susceptibilidade às infecções), maior sensibilidade à toxicidade por metais pesados e alta morbi-mortalidade (a anemia severa causa danos à regulação térmica) (UNICEF/UNU/WHO/MI, 1999). Alguns estudos sugerem, ainda, que as crianças anêmicas exibem um retardo no desenvolvimento neuromotor que não se modifica, mesmo após tratamento prolongado (Giugliani \& Victora, 1997; Lozzof et al., 1987). Por outro lado, existem evidências de que as crianças anêmicas tratadas com sulfato ferroso não só melhoraram a condição hematológica, como também apresentaram aumento do desenvolvimento psicomotor, além da melhoria da velocidade de crescimento pondo-estatural e do rendimento escolar (Aukett et al., 1986; Chwang et al., 1988; Pollitt, 1988).

No Brasil, não se pode ainda delinear um quadro sobre a distribuição e a prevalência da anemia na população, pois, apesar de diversas pesquisas executadas em muitas localidades do país, poucos foram os estudos com amostras representativas e que seguiram as recomendações da Organização Mundial da Saúde (OMS) no tocante aos procedimentos técnicos de coleta de dados, de análise laboratorial e de pontos de corte (Batista Filho, 1999).

Os primeiros dados publicados acerca da anemia em escolares, no Brasil, datam de 1945, oriundos da Pesquisa sobre Suplementação $\mathrm{Nu}$ tritiva em Escolares, realizada na cidade de Salvador por Azevedo e Galvão e descrita, em 1946, por Josué de Castro, em sua obra Geografia da Fome. O autor refere-se à anemia como "a mais generalizada" e "patente" das carências minerais, pois foi diagnosticada uma prevalência de $40 \%$ entre os escolares avaliados, que foi reduzida a 3,5\% após um tratamento com ferro, cálcio e vitaminas (Castro, 2001).

$\mathrm{Na}$ Região Nordeste, foram identificados poucos estudos sobre anemia em escolares. $\mathrm{Na}$ última década podem ser citados Ferreira et al. (1998), que avaliaram em 1997 um grupo de 299 escolares de 4 a 18 anos em São Lourenço da Mata, Pernambuco, registrando 43,1\% de anêmicos. Em Aracaju, capital do vizinho Estado de Sergipe, 354 escolares de 4 a 24 anos foram estudados em 1998, encontrando-se uma prevalência de $26,7 \%$ de anemia (Tsuyuoka et al., 1999). Não foram encontradas referências à situação da anemia no Estado de Alagoas na literatura específica.

O propósito deste estudo foi determinar a prevalência da anemia ferropriva e analisar a sua associação com o retardo pondo-estatural, em uma amostra representativa de escolares da primeira série do ensino fundamental, da rede pública de Maceió, Alagoas.

\section{Material e métodos}

A amostra foi planejada para representar o universo de 21.434 escolares matriculados no ano 2000, nas 541 turmas da primeira série do ensino fundamental, em 80 escolas estaduais e 45 municipais da cidade de Maceió, Estado de Alagoas. Na ausência de uma estimativa da prevalência da anemia ferropriva, e não sendo viável a realização de um projeto-piloto, adotou-se para o cálculo do tamanho da amostra, o procedimento que não requer informação prévia sobre 
o evento a ser estimado. Para um nível de confiança de 95\% e erro de estimação não superior a $5 \%$, calculou-se um $n=384$, mas prevendo possíveis perdas, este valor foi aumentado em cerca de $10 \%$, chegando-se a um $n=400$ escolares.

Na seleção da amostra empregou-se o processo de amostragem probabilística de estágios múltiplos em três etapas: seleção das escolas, das turmas e finalmente dos escolares. Em cada etapa, as unidades amostrais pertinentes foram enumeradas e o sorteio foi efetuado utilizandose o programa Epi-Info versão 6 (CDC, 1996). Esse procedimento resultou na seleção de 22 escolas (17 estaduais e 5 municipais) e de 426 crianças.

A coleta de dados foi realizada por uma equipe previamente treinada pela pesquisadora principal, no período de 25 de maio a 7 de julho de 2000.

O sangue foi colhido por venipuntura, com material descartável. Para a dosagem de hemoglobina, foi realizado o hemograma completo pelo contador eletrônico do tipo Coulter STKS, do Laboratório Central (LACEN - Alagoas), operado por técnicos treinados daquela instituição, cujo controle de qualidade é monitorado mensalmente pela Sociedade Brasileira de Análises Clínicas. A anemia ferropriva foi definida mediante dois pontos de corte para a hemoglobina, ambos propostos pela OMS: (1) a recomendação mais atual, que corresponde $\mathrm{a} \mathrm{Hb}<11,5 \mathrm{~g} /$ dl, para crianças na faixa etária de 5 a 11 anos (UNICEF/UNU/WHO/MI, 1999); (2) a indicação anterior que era $\mathrm{Hb}<12,0 \mathrm{~g} / \mathrm{dl}$ para escolares de 6 a 14 anos, que vigorou até outubro de 1998 (DeMaeyer \& Adiels-Tegman, 1985). Tornou-se necessário classificar a anemia por ambos os critérios, uma vez que a maioria das pesquisas anteriormente publicadas empregou o ponto de corte de $12,0 \mathrm{~g} / \mathrm{dl}$. Foi prescrito e fornecido sulfato ferroso a todas as crianças com $\mathrm{Hb}$ inferior a $12,0 \mathrm{~g} / \mathrm{dl}$.

Peso e estatura foram coletados conforme as técnicas sugeridas pela OMS; para o peso empregou-se uma balança Filizola antropométrica, capacidade $150 \mathrm{~kg}$, divisão em $100 \mathrm{~g}$ e para estatura o antropômetro portátil da marca Leicester (International Child Growth Foundation, Londres, Reino Unido). Os dados antropométricos foram analisados com o programa Epinut, um dos módulos do Epi-Info versão 6 (CDC, 1996), que calcula os indicadores $\mathrm{P} / \mathrm{I}, \mathrm{A} / \mathrm{I}$ e P/A, sendo o último indicador apenas para meninos até 138 meses de idade e $145 \mathrm{~cm}$ de altura e meninas até 120 meses e $137 \mathrm{~cm}$ de altura. Considerou-se o ponto de corte abaixo de -2,0 desvios-padrão da referência do National Center for Health Statistics (NCHS) (WHO, 1983).
Para a análise estatística empregou-se também o programa Epi-Info versão 6 (CDC, 1996). $\mathrm{Na}$ associação entre variáveis, usou-se o teste do qui-quadrado de Pearson e, quando apropriado, o teste de probabilidade exata de Fisher, sempre adotando o nível de significância de $5 \%$. Para as prevalências foram calculadas estimativas pontuais e intervalares, estas correspondendo ao intervalo de confiança de $95 \%$.

Definiu-se como critério de exclusão a presença de hemoglobina $S$, defeito genético que leva à anemia falciforme e que pode reduzir o nível de hemoglobina a valores críticos em portadores homozigóticos (Failace, 1995; Lorenzi, 1999; Verrastro et al., 1996). Tal diagnóstico foi realizado por meio da técnica da gelcentrifugação, posteriormente confirmado pela eletroforese de hemoglobina. Foram diagnosticados cinco casos de portadores do caráter falciforme, que foram devidamente documentados e encaminhados ao setor de Hematologia do Hospital Universitário da Universidade Federal de Alagoas (UFAL), a fim de receberem tratamento e acompanhamento adequados.

O Protocolo de Pesquisa foi aprovado pela Comissão de Ética em Pesquisa do Instituto Materno Infantil de Pernambuco (IMIP) e pela Comissão de Ética da UFAL. Após os devidos esclarecimentos os pais ou responsáveis assinaram o "Termo de consentimento livre e esclarecido" permitindo a participação de seus filhos na pesquisa.

\section{Resultados e discussão}

Do total de 426 escolares da amostra, 50,2\% eram do sexo masculino e $49,8 \%$ do sexo feminino. A idade variou entre seis anos e dez anos e dez meses, com uma média de 8,0 anos (desviopadrão $=1,0$ /ano). As principais características sociais e econômicas das famílias dos escolares incluídos no estudo estão apresentadas na Tabela 1 . Cerca de $20 \%$ dos pais e das mães eram analfabetos e em torno de $60 \%$ não completaram o lo grau. A ocupação dos pais mais referida foi o trabalho braçal $(25,2 \%)$, enquanto a grande maioria das mães não trabalhava fora de casa $(61,1 \%)$. A renda familiar auferida não ultrapassava a faixa de três salários mínimos em $86,4 \%$ das famílias.

De acordo com o novo ponto de corte $(\mathrm{Hb}<$ $11,5 \mathrm{~g} / \mathrm{dl}$ ), a anemia esteve presente em $9,9 \%$ dos escolares (IC 95\%: 7,3-12,9), mas segundo o ponto de corte tradicional $(\mathrm{Hb}<12,0 \mathrm{~g} / \mathrm{dl})$, a prevalência detectada neste grupo de crianças foi de 25,4\% (IC 95\%: 21,4-29,6) (Tabela 2). Todos os casos diagnosticados de anemia foram classifica- 
Tabela 1

Características sociais e econômicas das famílias de escolares da primeira série do ensino fundamental da rede pública. Maceió, Alagoas, Brasil, 2000.

\begin{tabular}{|c|c|c|c|c|}
\hline & \multicolumn{2}{|c|}{ Pai } & \multicolumn{2}{|c|}{ Mãe } \\
\hline & $\mathrm{n}$ & $\%$ & $\mathrm{n}$ & $\%$ \\
\hline \multicolumn{5}{|l|}{$\begin{array}{l}\text { Nível de escolaridade dos pais } \\
\text { de escolares incluídos no estudo }\end{array}$} \\
\hline Analfabeto & 70 & 19,6 & 85 & 20,3 \\
\hline Ensino fundamental incompleto & 208 & 58,2 & 258 & 61,7 \\
\hline Ensino fundamental completo & 42 & 11,8 & 35 & 8,4 \\
\hline Ensino médio incompleto & 12 & 3,4 & 17 & 4,1 \\
\hline Ensino médio completo & 25 & 7,0 & 23 & 5,5 \\
\hline Total & 357 & 100,0 & 418 & 100,0 \\
\hline \multicolumn{5}{|l|}{$\begin{array}{l}\text { Ocupação dos pais de escolares } \\
\text { incluídos no estudo }\end{array}$} \\
\hline Trabalhador braçal & 99 & 25,2 & - & - \\
\hline Trabalhador Indústria/Comércio & 49 & 12,5 & 25 & 5,9 \\
\hline Condutor & 30 & 7,7 & - & - \\
\hline Vendedor(a) & 29 & 7,4 & 14 & 3,3 \\
\hline Vigilante & 27 & 6,9 & - & - \\
\hline Servente & 13 & 3,3 & 85 & 20,0 \\
\hline Dona de casa & - & - & 260 & 61,1 \\
\hline Desempregado(a) & 48 & 12,2 & 19 & 4,5 \\
\hline Outras & 97 & 24,8 & 22 & 5,2 \\
\hline Total & 392 & 100,0 & 425 & 100,0 \\
\hline \multicolumn{5}{|l|}{$\begin{array}{l}\text { Renda familiar mensal das famílias } \\
\text { de escolares incluídos no estudo } \\
(\mathrm{SM}=\mathrm{R} \$ 151,00)\end{array}$} \\
\hline$<1,0 \mathrm{SM}$ & \multicolumn{2}{|c|}{61} & \multicolumn{2}{|c|}{14,6} \\
\hline $1,0-1,4 \mathrm{SM}$ & \multicolumn{2}{|c|}{143} & \multicolumn{2}{|c|}{34,2} \\
\hline $1,5-1,9 \mathrm{SM}$ & \multicolumn{2}{|c|}{66} & \multicolumn{2}{|c|}{15,8} \\
\hline $2,0-2,4 \mathrm{SM}$ & \multicolumn{2}{|c|}{54} & \multicolumn{2}{|c|}{12,9} \\
\hline $2,5-2,9 \mathrm{SM}$ & \multicolumn{2}{|c|}{37} & \multicolumn{2}{|c|}{8,9} \\
\hline$\geq 3,0 \mathrm{SM}$ & \multicolumn{2}{|c|}{57} & \multicolumn{2}{|c|}{13,6} \\
\hline Total & \multicolumn{2}{|c|}{418} & \multicolumn{2}{|c|}{100,0} \\
\hline
\end{tabular}

$\mathrm{SM}=$ salário mínimo.

Tabela 2

Classificação da anemia segundo a taxa de hemoglobina em escolares da primeira série do ensino fundamental da rede pública. Maceió, Alagoas, Brasil, 2000.

\begin{tabular}{lrrrr}
\hline Classificação & \multicolumn{3}{c}{ Hemoglobina } & \multicolumn{2}{c}{$<12,0 \mathrm{~g} / \mathrm{d}$ lb } \\
& \multicolumn{1}{c}{$\mathrm{n}$} & $\%$ & $\mathrm{n}$ & $\%$ \\
\hline Anemia & 42 & 9,9 & 108 & 25,4 \\
Normalidade & 384 & 90,1 & 318 & 74,6 \\
Total & 426 & 100,0 & 426 & 100,0 \\
\hline
\end{tabular}

a UNICEF/UNU/WHO/MI (1999)

b DeMaeyer \& Adiels-Tegman (1985). dos nas formas "leve" e "moderada", visto que o menor valor de hemoglobina encontrado foi de 9,3g/dl.

A recente proposta de alteração do ponto de corte pela OMS, evidentemente, embasou-se em critérios epidemiológicos e deverá ser usada doravante nas pesquisas com população nesta faixa etária. No caso específico deste estudo, a mudança do ponto de corte em $0,5 \mathrm{~g} / \mathrm{dl}$ de hemoglobina acarretou uma alteração na prevalência de anemia em mais de $100 \%$ : pelo critério tradicional, mais de um quarto dos escolares estudados deveria ser considerado anêmico, valor que passou a menos de $10 \%$, excluindo os prováveis "falsos positivos" do critério anterior.

A distribuição dos valores de hemoglobina na população estudada está ilustrada na Figura 1. A curva apresenta um formato que se aproxima ao de uma distribuição normal; o valor médio foi de $12,4 \mathrm{~g} / \mathrm{dl}$ (desvio-padrão $=0,8 \mathrm{~g} / \mathrm{dl}$ ).

Dentre os estudos epidemiológicos realizados com escolares no Brasil, apenas dois classificaram a anemia pelo novo critério de $\mathrm{Hb}<$ $11,5 \mathrm{~g} / \mathrm{dl}$. O primeiro se refere a uma avaliação com escolares de 6 a 12 anos de idade, procedentes de famílias de baixa renda de uma comunidade rural do Paraná; a prevalência de anemia encontrada entre essas crianças foi de $25,7 \%$ (Sichieri et al., 1996). A outra pesquisa diz respeito à investigação de escolares de 6 a 11 anos em instituições municipais de ensino na área urbana do Rio de Janeiro, onde a anemia foi encontrada em 13,0\% das crianças estudadas (SMSRJ/SME-RJ, 2000). Os resultados alcançados nos estudos realizados em zonas urbanas foi bastante semelhante: 9,9\% entre os escolares de Maceió e 13,0\% entre as crianças do Rio de Janeiro. Diferem do achado da pesquisa do Paraná $(25,7 \%)$ porque, provavelmente, a anemia segue o mesmo padrão de outras carências nutricionais, quase sempre mais freqüentes em áreas rurais do que em urbanas.

Já a anemia avaliada pelo ponto de corte de $\mathrm{Hb}<12,0 \mathrm{~g} / \mathrm{dl}$ é o critério utilizado pela maioria dos trabalhos publicados nessa área de estudo; a prevalência do problema entre escolares no Brasil é bastante variada, mas quase sempre de alta magnitude. Tsuyuoka et al. (1999), estudando escolares do ensino público em Aracaju, encontraram $\mathrm{Hb}<12,0 \mathrm{~g} / \mathrm{dl}$ em $26,7 \%$ das crianças avaliadas, verificando, ainda, que a proporção de anêmicos era maior entre os menores de 8 anos e entre os maiores de 15 anos de idade. Stefanini et al. (1995), estudando alunos ingressantes nas primeiras séries do ensino fundamental das escolas públicas de Osasco, identificaram a anemia ferropriva $(\mathrm{Hb}<12,0 \mathrm{~g} / \mathrm{dl})$ em $51,0 \%$ das crianças investigadas. Um traba- 
Distribuição dos níveis de hemoglobina na amostra de escolares da primeira série do ensino fundamental da rede pública. Maceió, Alagoas, Brasil, 2000.

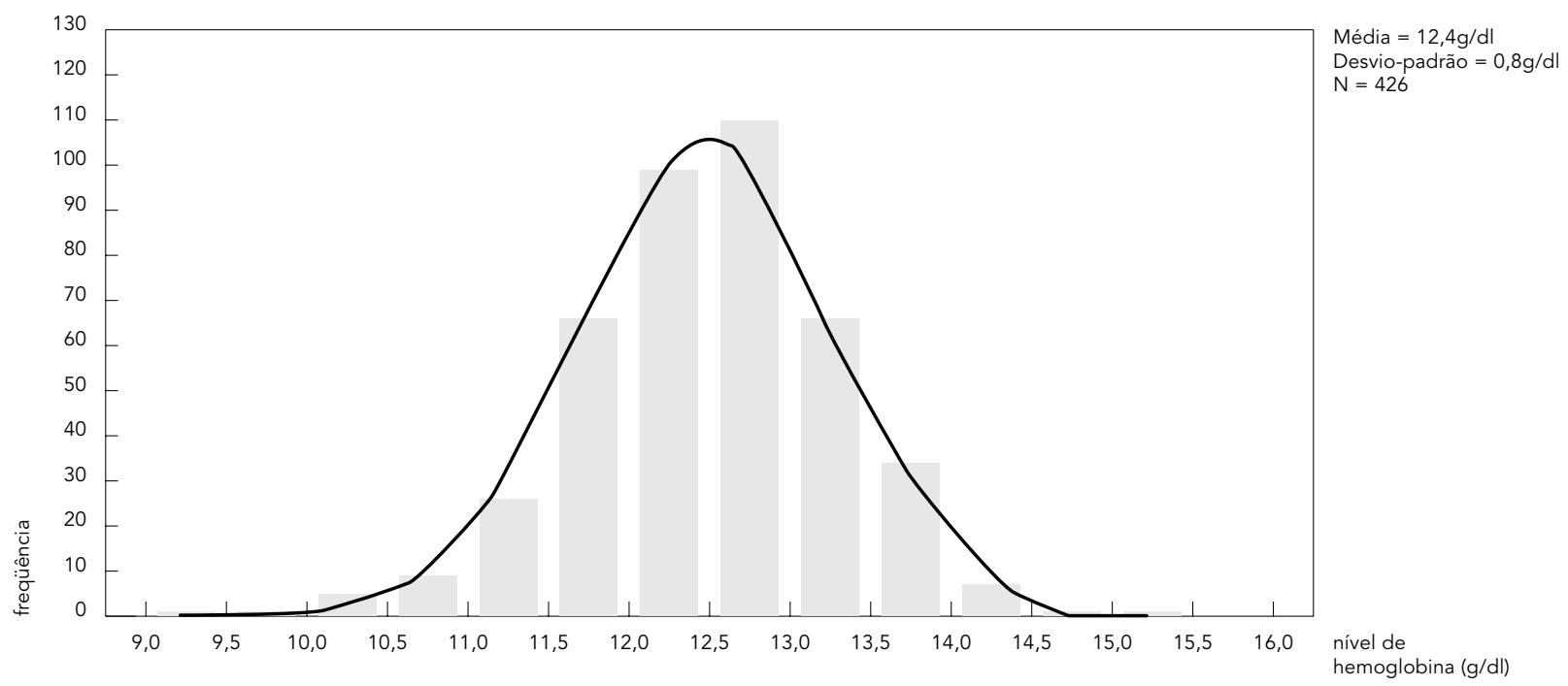

lho realizado na área rural de Pernambuco (Ferreira et al., 1998), avaliando uma população de 4 a 18 anos de idade, diagnosticou anemia $(\mathrm{Hb}<$ $12,0 \mathrm{~g} / \mathrm{dl}$ ) em $43,1 \%$ dos escolares examinados. Os resultados encontrados nesta população de escolares de Maceió $(25,4 \%$ com $\mathrm{Hb}<12,0 \mathrm{~g} / \mathrm{dl})$ se encontram dentro da faixa de variação dos estudos citados.

A distribuição da prevalência de anemia, classificada pelos dois pontos de corte entre as crianças eutróficas e aquelas com retardo pondo-estatural (abaixo de -2,0 DP), não alcançou significância estatística tanto em relação aos indicadores altura para idade (A/I), como peso para idade $(\mathrm{P} / \mathrm{I})$ e peso para altura $(\mathrm{P} / \mathrm{A})$ (Tabela 3 e Tabela 4).

Não houve associação entre a anemia ferropriva e o retardo do crescimento diagnosticados nos escolares alagoanos. O resultado desta pesquisa corrobora os encontrados nos trabalhos de Stefanini et al. (1995) e Sichieri et al. (1996), que, embora referindo alta prevalência de anemia nos escolares estudados, não encontraram relação estatisticamente significativa entre retardo pondo-estatural e anemia.

A presente pesquisa confirma, portanto, dois achados importantes que vêm sendo relatados em inquéritos nutricionais recentes com crianças pré-escolares no Brasil: prevalências elevadas de anemia que superam, de longe, as preva- lências do retardo pondo-estatural e ausência de associação entre esses dois tipos de desnutrição. Há que ressaltar os resultados dos estudos de série temporal que mostram uma tendência de aumento significativo da anemia em crianças de 0 a 60 meses: um conduzido no Município de São Paulo (Monteiro et al., 2000) e outro no Estado da Paraíba (Oliveira et al., 2002). Em São Paulo a prevalência da anemia passou de $22,7 \%$ (1973-1974), para 35,6\% (1984-1985) e 46,9\% (1995-1996); já na Paraíba o incremento em dez anos foi de 19,3\% (IC 95\%: 7,3-21,5) (1982) para 36,4\% (IC 95\%: 33,7-39,1) (1992). Os estudos com escolares são escassos e não existe nenhum acompanhamento de série temporal que permita verificar se neste grupo populacional a tendência de elevação da anemia também ocorre.

\section{Conclusões}

A deficiência de ferro representa um espectro de situações carenciais que culmina com a manifestação da anemia ferropriva. Em uma população normal a variação esperada dos valores de hemoglobina gera uma curva de distribuição, em que cerca de $2,5 \%$ dos indivíduos apresentam valores de $\mathrm{Hb}$ inferior aos pontos de corte propostos (cerca de dois desvios-padrão abaixo da média); ao superar o limite de $5 \%$, contu- 
Distribuição dos níveis de hemoglobina abaixo de 11,5g/dl, segundo os indicadores antropométricos, nos escolares da primeira série do ensino fundamental da rede pública. Maceió, Alagoas, Brasil, 2000.

\begin{tabular}{|c|c|c|c|c|c|c|c|c|c|c|c|c|}
\hline \multirow{4}{*}{$\begin{array}{l}\text { Nível de } \\
\text { hemoglobina } \\
\text { (g/dl) }\end{array}$} & \multicolumn{12}{|c|}{ Indicadores antropométricos } \\
\hline & \multicolumn{4}{|c|}{$A / / a$} & \multicolumn{4}{|c|}{$P / I b$} & \multicolumn{4}{|c|}{$P / A c$} \\
\hline & \multicolumn{2}{|c|}{ Deficiente } & \multicolumn{2}{|c|}{ Eutrófico } & \multicolumn{2}{|c|}{ Deficiente } & \multicolumn{2}{|c|}{ Eutrófico } & \multicolumn{2}{|c|}{ Deficiente } & \multicolumn{2}{|c|}{ Eutrófico } \\
\hline & $\mathrm{n}$ & $\%$ & $\mathrm{n}$ & $\%$ & $\mathrm{n}$ & $\%$ & $n$ & $\%$ & $\mathrm{n}$ & $\%$ & $\mathrm{n}$ & $\%$ \\
\hline $\mathrm{Hb}<11,5$ & 4 & 15,4 & 38 & 9,5 & 3 & 18,8 & 39 & 9,5 & 0 & 0,0 & 42 & 10,5 \\
\hline $\mathrm{Hb} \geq 11,5$ & 22 & 84,6 & 362 & 90,5 & 13 & 81,2 & 371 & 90,5 & 12 & 100,0 & 358 & 89,5 \\
\hline Total & 26 & 100,0 & 400 & 100,0 & 16 & 100,0 & 410 & 100,0 & 12 & 100,0 & 400 & 100,0 \\
\hline
\end{tabular}

a $p=0,308$

b $p=0,202$

c $p=0,621$

Tabela 4

Distribuição dos níveis de hemoglobina, abaixo de $12,0 \mathrm{~g} / \mathrm{dl}$ segundo os indicadores antropométricos, nos escolares da primeira série do ensino fundamental da rede pública. Maceió, Alagoas, Brasil, 2000.

\begin{tabular}{|c|c|c|c|c|c|c|c|c|c|c|c|c|}
\hline \multirow{3}{*}{$\begin{array}{l}\text { Nível de } \\
\text { hemoglobina } \\
\text { (g/dl) }\end{array}$} & \multicolumn{12}{|c|}{ Indicadores antropométricos } \\
\hline & \multicolumn{2}{|c|}{ Deficiente } & \multicolumn{2}{|c|}{ Eutrófico } & \multicolumn{2}{|c|}{ Deficiente } & \multicolumn{2}{|c|}{ Eutrófico } & \multicolumn{2}{|c|}{ Deficiente } & \multicolumn{2}{|c|}{ Eutrófico } \\
\hline & $\mathrm{n}$ & $\%$ & $\mathrm{n}$ & $\%$ & $\mathrm{n}$ & $\%$ & $\mathrm{n}$ & $\%$ & $\mathrm{n}$ & $\%$ & $\mathrm{n}$ & $\%$ \\
\hline $\mathrm{Hb}<12,0$ & 9 & 34,6 & 99 & 24,8 & 5 & 31,2 & 103 & 25,1 & 1 & 8,3 & 104 & 26,0 \\
\hline $\mathrm{Hb} \geq 12,0$ & 17 & 65,4 & 301 & 75,2 & 11 & 68,8 & 307 & 74,9 & 11 & 91,7 & 296 & 74,0 \\
\hline Total & 26 & 100,0 & 400 & 100,0 & 16 & 100,0 & 410 & 100,0 & 12 & 100,0 & 400 & 100,0 \\
\hline
\end{tabular}

a $p=0,262$

b $p=0,565$

c $p=0,310$

do, já se pode considerar que existe anemia numa dada população (UNICEF/UNU/WHO/MI, 1999). Esta prevalência crítica de $5 \%$ foi ultrapassada nesta pesquisa, mesmo quando se leva em conta o ponto de corte mais restritivo de $\mathrm{Hb}<11,5 \mathrm{~g} / \mathrm{dl}$. Logo, os resultados demonstram que o problema é sério e merece atenção.

Frente à elevada prevalência encontrada, justificam-se políticas públicas de largo alcance para o enfrentamento do problema. Uma dessas estratégias foi posta em prática recentemen-

\section{Agradecimentos}

À Fundação de Amparo à Pesquisa do Estado de Alagoas, pelo financiamento do estudo; ao Laboratório Central/Alagoas e sua equipe, pela realização dos exames hematológicos; aos dirigentes, professores e funcionários das 22 escolas; aos escolares e seus pais; às acadêmicas de Nutrição e entrevistadoras: Anna L. Ferreira, Célia Batista, Daniela Araújo, Fabiana Rios, Kalline Oliveira, Karla Rocha, Thaísa Vilanova e Vanessa te: a fortificação de farinhas de trigo e de milho com ferro tornar-se-á obrigatória no Brasil por Portaria Ministerial (Consulta Pública no 51 da Agência Nacional de Vigilância Sanitária do Ministério da Saúde) (MS, 2002) e se tornará efetiva a partir de 2003. Todavia, a curto prazo, é necessário implementar outras medidas, como forma de benefício dirigido especialmente à criança nessa faixa etária, a exemplo da inclusão de fontes de ferro de alta biodisponibilidade na merenda escolar.
Prudente; às profissionais que coletaram sangue: Sandra C. Cavalcante e Maria José Silva e aos colaboradores Maurício Pereira e Antônio Veiga; ao Prof. Celso Tavares, pela prescrição do tratamento para as crianças; ao Prof. Malaquias Batista Filho e ao Instituto Materno Infantil de Pernambuco pelo apoio e fornecimento do sulfato ferroso. A todos, os nossos agradecimentos, pela inestimável participação. 


\section{Referências}

AUKETT, M. A.; PARKS, Y. A.; SCOTT, P. H. \&WHARTON, B. A., 1986. Treatment with iron increases weight gain and psycomotor development. Archives of Disease in Childhood, 61:849-857.

BATISTA FILHO, M., 1999. Saúde e nutrição. In: Epidemiologia e Saúde (M. Z. Rouquayrol, org.), pp. 353374, 5a Ed. Rio de Janeiro: Medsi.

CASTRO, J., 2001. Geografia da Fome. O Dilema Brasileiro: Pão ou Aço. 14a Ed. Rio de Janeiro: Civilização Brasileira.

CDC (Centers for Disease Control and Prevention)/ WHO (World Health Organization), 1996. Epi Info 6, Version 6.04. A Word Processing, Database, and Statistics Program for Public Health. Atlanta: CDC/ Geneva: WHO.

CHWANG, L.; SOEMANTRI, A. G. \& POLLITT, E., 1988. Iron supplementation and physical growth of rural Indonesian children. American Journal of Clinical Nutrition, 47:496-501.

DeMAEYER, E. M. \& ADIELS-TEGMAN, M., 1985. The prevalence of anaemia in the world. World Health Statistics Quarterly, 38:302-316.

FAILACE, R., 1995. Hemograma: Manual de Interpretação. Porto Alegre: Artes Médicas.

FERREIRA, L. O. C., 1998. A Ação do Sulfato Ferroso Administrado em Doses Diárias e Semanais em Escolares da Mata Sul de Pernambuco: Um Ensaio Terapêutico. Tese de Doutorado, Recife: Centro de Ciências da Saúde, Universidade Federal de Pernambuco.

FERREIRA, M. R.; SOUZA, W.; PEREZ, E. P.; LAPA, T.; CARVALHO, A. B.; FURTADO, A.; COUTINHO, H. B. \& WAKELIN, D., 1998. Intestinal helminthiasis and anaemia in youngsters from Matriz da Luz, district of São Lourenço da Mata, State of Pernambuco, Brazil. Memórias do Instituto Oswaldo Cruz, 93:289-293.

GIUGLIANI, E. R. J. \& VICTORA, C. G., 1997. Normas Alimentares para Crianças Brasileiras Menores de Dois Anos: Bases Científicas. Brasília: Organização Pan-Americana da Saúde/Organização Mundial da Saúde.

LORENZI, F. T., 1999. Manual de Hematologia: Propedêutica e Clínica. Rio de Janeiro: Editora Médica e Científica.

LOZZOF, B.; BRITTENHAM, G. M.; WOLF, A. W.; McCLISH, D. K.; KUHNERT, P. M.; JIMENEZ, R.; MORA, L. A.; GOMEZ, I. \& KRAUSKOPH, D., 1987. Iron deficiency anemia and iron therapy effects on infant development tests performance. Pediatrics, 79:981-995.

MONTEIRO, C. A.; SZARFARC, S. C. \& MONDINI, L., 2000. Tendência secular da anemia na infância, na cidade de São Paulo (1984-1996). Revista de Saúde Pública, 34:62-72.
MS (Ministério da Saúde), 2002. Consulta Pública no 51 de 10 de junho de 2002. Regulamento Técnico para Fortificação com Ferro e Ácido Fólico nas Farinhas de Trigo e nos Derivados do Milho. 10 Junho 2002 <http://www.anvisa.gov.br/consulta/2002/ 51_2002.htm>.

OLIVEIRA, R. S.; DINIZ, A. S.; BENIGNA, M. J. C. N.; SILVA, S. M. M.; LOLA, M. M. F.; GONÇALVES, M. C.; MOURA, L. A.; RIVERA, M. A. A. \& SANTOS, L. M. P., 2002. Magnitude, distribuição espacial e tendência da anemia em pré-escolares da Paraíba. Revista de Saúde Pública, 36:26-32.

POLLITT, E., 1988. Desnutrición y rendimiento escolar. In: Crecimiento y Desarrollo: Hechos y Tendencias (M. Cusminsky, E. M. Moreno \& E. N. S. Ojeda, org.), Publicación Científica 510, pp. 324-348, Washington, DC: Organización Mundial de la Salud/Organización Panamericana de la Salud.

SMS-RJ (Secretaria Municipal de Saúde do Rio de Janeiro)/SME-RJ (Secretaria Municipal de Educação do Rio de Janeiro), 2000. II Pesquisa de Saúde e Nutrição em Escolares. Rio de Janeiro: Prefeitura Municipal do Rio de Janeiro.

SICHIERI, R.; MATHIAS, T. \& MOURA, A. S., 1996. Stunting, high weight-for-height, anemia e dietary intake among brazilian students from a rural community. Nutrition Research, 16:201-209.

STEFANINI, M. L. R.; COLLI, C.; LERNER, B. R.; LEI, D. L. M.; CHAVES, S. P.; DI PIETRO, M. S.; OLIVEIRA, A. A. M. \& SZARFAC, S. C., 1995. Anemia e desnutrição em escolares da rede pública do Município de Osasco, São Paulo, Brasil. Cadernos de Saúde Pública, 11:439-447.

TSUYUOKA, R.; BAILEY, J. W.; GUIMARÃES, A. M. A. N.; GURGEL, R. Q. \& CUEVAS, L. E., 1999. Anemia and intestinal parasitic infections in primary school students in Aracaju, Sergipe, Brazil. Cadernos de Saúde Pública, 15:413-421.

UNICEF (United Nations Children's Fund)/UNU (United Nations University)/WHO (World Health Organization)/MI (Micronutrient Iniciative). 1999. Preventing Iron Deficiency in Women and Children. Technical Workshop. Boston: International Nutrition Foundation.

VERRASTRO, T.; LORENZI, T. \& WENDEL NETO, S., 1996. Hematologia e Hemoterapia: Fundamentos de Morfologia, Fisiologia, Patologia e Clínica. São Paulo: Atheneu.

WHO (World Health Organization), 1983. Measuring Change in Nutritional Status: Guidelines for Assessing the Nutritional Impact of Supplementary Feeding Programs for Vulnerable Groups. Geneva: WHO.

Recebido em 23 de novembro de 2001

Versão final reapresentada em 26 de março de 2002 Aprovado em 18 de junho de 2002 